\title{
Matrice de transfert et sources hydroacoustiques d'une pompe centrifuge à faibles charges
}

\author{
Transmission matrix and hydroacoustic sources \\ of a centrifugal pump at very low load
}

\author{
par J.-F. Lauro, A. Boyer \\ EDF/DER/Département Machines
}

Transmission matrix and hydroacoustic sources of a centrifugal pump, at very low load, are determined by an experimental method; with an external excitation (flow fluctuations generated by a small rotating valve connected on a by-pass around the machine), more important than the hydroacoustic sources of the pump (even at very low load), the transmission matrix is accurately determined. Then pump sources are obtained by a simple difference. The main results are:

- in terms of load, no variation of the transmission matrix,

- about hydroacoustic sources, under a particular load (beginning of recirculation phenomena), spectra are distorted and the peak amplitude at the blade passing frequency (and multiples) increases appreciably, - at low load, wide band noise becomes important $(x 20)$, and this has a very bad influence on arounding pipes, because their hydroacoustic modes are necessarily excited.

\section{I 口 INTRODUCTION}

Les circuits industriels parcourus par des fluides sont le siège de phénomènes vibratoires résultant des fluctuations de pression internes dues aux écoulements. Ces perturbations sont de deux types :

- le premier est appelé fluctuations de pression acoustique ou bruit, de faible amplitude, irrotationnel et se propage à la vitesse du son dans tout le circuit,

- le second type (appelé pseudo-bruit) est un phénomène dû à la turbulence, il est non propagatif et convecté par les structures de l'écoulement.

Bien que l'énergie des fluctuations acoustiques soit faible par rapport à l'énergie mécanique du fluide, le couplage avec la structure peut être nuisible, surtout pour des écoulements dans les milieux industriels où l'on risque de voir apparaître des ruptures de piquages ou de matériel par fatigue.

Afin de prédire le comportement acoustique et vibratoire des circuits hydrauliques sous écoulement, la Direction des Etudes et Recherches d'EDF (Département Acoustique et Mécanique Vibratoire) a développé le code CIRCUS ( $\left.{ }^{1}\right)$.
L'utilisation de ce code nécessite la connaissance des caractéristiques hydroacoustiques des composants des circuits. Actuellement, dans la base de données de CIRCUS ${ }^{1}$, il existe des modèles acoustiques pour différentes singularités et en particulier les pompes centrifuges. Celles-ci ont toujours été étudiées autour de leur point nominal de fonctionnement. Cela semblait incomplet, et en voici un exemple.

Dans une centrale nucléaire, les pompes de sauvegarde sont testées à intervalles réguliers (environ une fois par mois), et pendant ces tests, la charge est souvent très faible (10\% environ). D'importants phénomènes acoustico-vibratoires peuvent alors apparaître entre la machine utilisée à une charge inhabituelle et les tuyauteries alentour.

Dans le but de pouvoir estimer, avec CIRCUS, les réponses acoustico-vibratoires du réseau de tuyauteries pendant ces fonctionnements particuliers, nous allons :

(1) CIRCUS : Logiciel calculant, pour un réseau de tuyauteries quelconque sous écoulement (1D), la réponse acoustico-vibratoire des tuyauteries à un chargement donné (calcul direct ou prédictif). CIRCUS peut être aussi utilisé en calcul inverse ou diagnostic, à partir de mesures de pression fluctuante sur les tuyauteries. 
- déterminer, par des méthodes expérimentales, la matrice de transfert et les sources hydroacoustiques d'une pompe centrifuge à basses charges,

- étudier et comprendre les différences notables avec les résultats obtenus autour de la charge nominale.

Ces essais se sont déroulés, en mai 97 , sur la boucle SESAME du Laboratoire de Mécanique de Lille dans les locaux de l'Ecole Nationale des Arts et Métiers de Lille.

\section{II —QUELQUES RAPPELS}

\subsection{Les modèles hydroacoustiques de type matrice/source}

On considère que la propagation des ondes se fait en ondes planes (on ne considère qu'une seule variable d'espace). Pour décrire le comportement hydroacoustique d'une partie d'un circuit hydraulique, on utilise une formulation matricielle. C'est sur ce principe qu'est basé CIRCUS. La relation entre les vecteurs d'état de deux points successifs d'une tuyauterie s'écrit :

$$
\left[\begin{array}{l}
p 2 \\
q 2
\end{array}\right]=[M] \bullet\left[\begin{array}{l}
p 1 \\
q 1
\end{array}\right]
$$

$p 1$ et $q 1:$ partie fluctuante de la pression et du débit au point 1.

$p 2$ et $q 2$ : partie fluctuante de la pression et du débit au point 2.

Il est possible de décrire par une telle formulation (en utilisant au besoin une succession de matrices) toutes les parties passives d'un circuit hydraulique. Dans le cas où le circuit comporte un composant actif, c'est-à-dire un composant qui génère des perturbations hydroacoustiques, il faut ajouter à la formulation un vecteur source. L'équation devient alors

$$
\left[\begin{array}{l}
p 2 \\
q 2
\end{array}\right]=[M] \cdot\left[\begin{array}{l}
p 1 \\
q 1
\end{array}\right]+\left[\begin{array}{l}
\Delta p s \\
\Delta q s
\end{array}\right]
$$

$\Delta p s$ : saut de fluctuation de pression.

$\Delta q s$ : saut de fluctuation de débit.

A partir de ces deux éléments (matrice de transfert et source), il est alors possible de décrire complètement le comportement hydroacoustique d'un circuit quelconque, à condition de disposer d'une représentation matricielle de chaque composant du circuit.

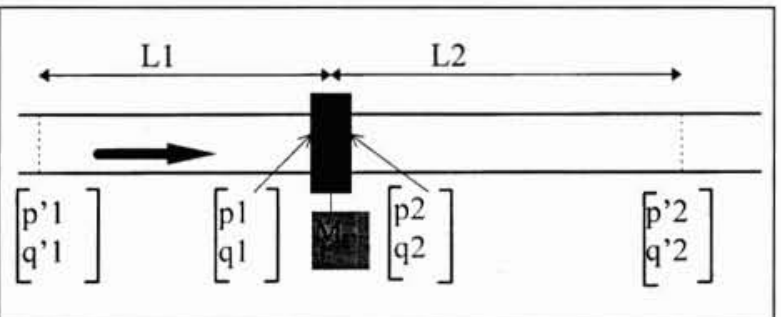

'1 position du capteur à l'amont de la singularité

(à une distance L1)

1 amont de la singularité

2 aval de la singularité

' 2 position du capteur à l'aval de la singularité

(à une distance L2).

1. Nécessité d'utiliser des matrices de transport en tube rectiligne pour établir les vecteurs d'état à l'amont et à l'aval de la singularité.
Dans le cas pratique, les mesures ne peuvent pas être effectuées au voisinage immédiat de la singularité. Il faut donc prendre en compte la distance de la mesure à la singularité et appliquer des matrices de transport (en tube rectiligne). Dans la suite, nous ne considérerons que des mesures ramenées à la singularité.

\subsection{Intensimétrie hydroacoustique}

En utilisant les formules de $p(x, f)$ et $q(x, f)$ dans le domaine fréquentiel, il est possible de déterminer la valeur de la célérité c. Il est utile d'avoir pour cette mesure un triplet de capteurs $p 1, p 2$ et $p 3$ équidistants. On a alors la formulation suivante :

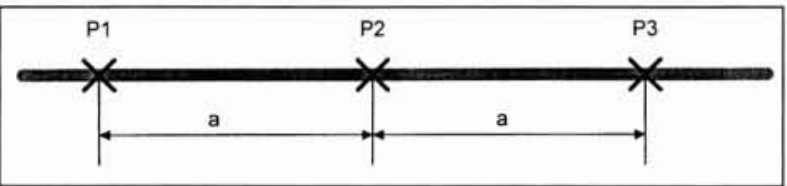

2. Implantation des capteurs pour un calcul intensimétrique.

$$
\frac{p_{1}(x, f)+p_{3}(x, f)}{p_{2}(x, f)}=\left(e^{-i k+a}+e^{+i k-a}\right)
$$

Si le nombre de Mach est faible, on obtient une fonction cosinus réelle :

$$
\frac{1}{2} \frac{p_{1}(x, f)+p_{3}(x, f)}{p_{2}(x, f)}=\cos \frac{\omega a}{c}
$$

La célérité c étant connue, on peut en déduire le nombre d'onde $k$ en fonction de la fréquence. Considérons nos trois capteurs équidistants précédents, ainsi qu'une abscisse $x=0$ pour $p 2$, alors $p 1$ sera situé à $x=-a$ et $p 3$ à $x=a$. On peut donc écrire :

$$
\begin{aligned}
& p_{1}=A(f) e^{+i k a}+B(f) e^{-i k a} \\
& p_{2}=A(f)+B(f) \\
& p_{3}=A(f) e^{-i k a}+B(f) e^{+i k a}
\end{aligned}
$$

Dans le cas où $k$ est considéré comme constant $(M \ll<1)$, 2 équations suffiraient pour connaître $A$ et $B$. La troisième permet de faire une approximation des moindres carrés.

$A(f), B(f)$ et $c$ sont alors connus en tout point d'implantation des capteurs. On peut donc calculer $p$ et $q$ en tout point de la veine d'essais, en utilisant la résolution de l'équation d'onde dans le domaine fréquentiel :

$$
\begin{gathered}
p(x, f)=A(f) \cdot e^{-i k x}+B(f) \cdot e^{+i k x} \\
q(x, f)=\frac{S}{\rho c} \cdot\left[A(f) \cdot e^{-i k x}-B(f) \cdot e^{+i k x}\right]
\end{gathered}
$$

$S$ : section de la tuyauterie $\rho$ : masse volumique du fluide

\subsection{Détermination de la matrice de transfert}

Afin de déterminer expérimentalement les quatre termes de la matrice de transfert d'une singularité, il est utile de mon- 


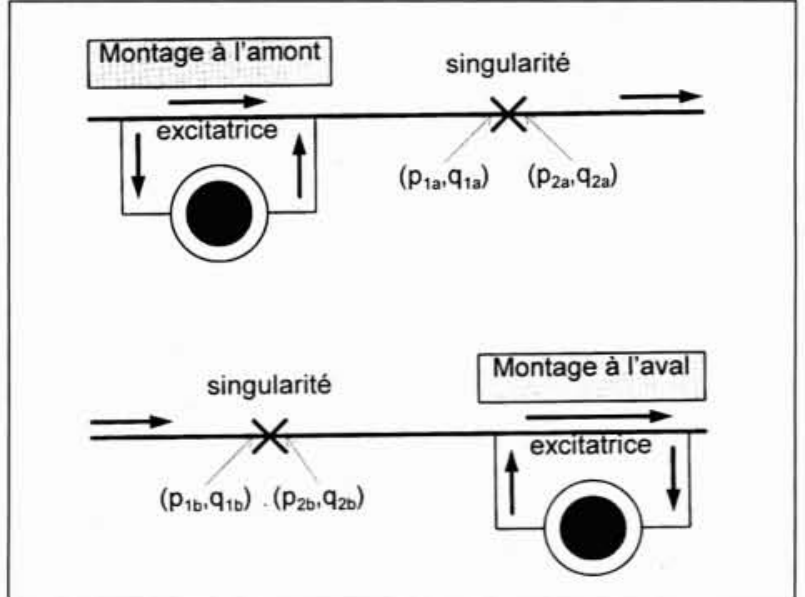

3. Les deux montages de l'excitatrice (amont et aval).

ter un système d'excitation dont les signaux seront plus importants que ceux du terme source. On réalise 2 essais $(a$ et $b)$ avec une source externe située à l'extrémité de la zone instrumentée, successivement à l'amont (essai $a$ ) puis à l'aval (essai $b$ ). On utilise pour cela une vanne tournante comme moyen d'excitation de débit et on vérifie que les sources externes sont nettement supérieures à la source générée par la pompe.

On peut donc écrire :

- Pour le montage amont : $\quad\left[\begin{array}{c}p 2 a \\ q 2 a\end{array}\right]=\left[\begin{array}{ll}a & b \\ c & d\end{array}\right] *\left[\begin{array}{c}p 1 a \\ q 1 a\end{array}\right]$

- Pour le montage aval :

$$
\left[\begin{array}{l}
p 2 b \\
q 2 b
\end{array}\right]=\left[\begin{array}{ll}
a & b \\
c & d
\end{array}\right] *\left[\begin{array}{l}
p 1 b \\
q 1 b
\end{array}\right]
$$

On obtient ainsi un système de 4 équations à 4 inconnues qui permet de connaître les 4 termes de la matrice de transfert.

\subsection{Détermination des termes sources}

Après la détermination de la matrice de transfert de la singularité, on peut évaluer les sources de débit avec un essai sans excitatrice dans les mêmes conditions d'écoulement. On résout alors le système d'équations suivant :

$$
\left[\begin{array}{l}
p_{2} \\
q_{2}
\end{array}\right]=\left[\begin{array}{ll}
a & b \\
c & d
\end{array}\right] *\left[\begin{array}{c}
p_{1} \\
q_{1}+\Delta q_{a s p}
\end{array}\right]+\left[\begin{array}{c}
0 \\
\Delta q_{r e f}
\end{array}\right]
$$

où les seules inconnues sont $\Delta q_{a s p}$ et $\Delta q_{r e f}$

Cette écriture, avec 2 sources de débit, est en accord avec le logiciel CIRCUS utilisé en calcul inverse (les données sont les enregistrements des capteurs et les résultats sont les sources des singularités). Cette écriture est équivalente à celle des 2 sources complètes (source de pression et source de débit) avec hypothèse de colinéarité.

On a :

$$
\begin{aligned}
& p_{2}=a \cdot p_{1}+b\left(q_{1}+\Delta q_{a s p}\right) \\
& q_{2}=c \cdot p_{1}+d\left(q_{1}+\Delta q_{a s p}\right)+\Delta q_{r e f} \\
& \qquad q_{a s p}=\left(p_{2}-a \cdot p_{1}\right) / b-q_{1} \\
& \Delta q_{r e f}=q_{2}-c \cdot p_{1}-d\left(p_{2}-a \cdot p_{1}\right) / b
\end{aligned}
$$

\section{LA BOUCLE SESAME}

Pour ces essais, la pompe étudiée a été installée sur la boucle SESAME du Laboratoire de Mécanique de Lille, située dans l'Ecole Nationale Supérieure des Arts et Métiers de Lille. Elle est fixée entre 2 réservoirs (voir le schéma cidessous). Le réglage du débit est assuré par plusieurs vannes, ce qui permet de réduire les bruits parasites. La tuyauterie d'aspiration a un diamètre de $250 \mathrm{~mm}$ et la tuyauterie de refoulement de $200 \mathrm{~mm}$. Ces 2 tuyauteries sont équi-

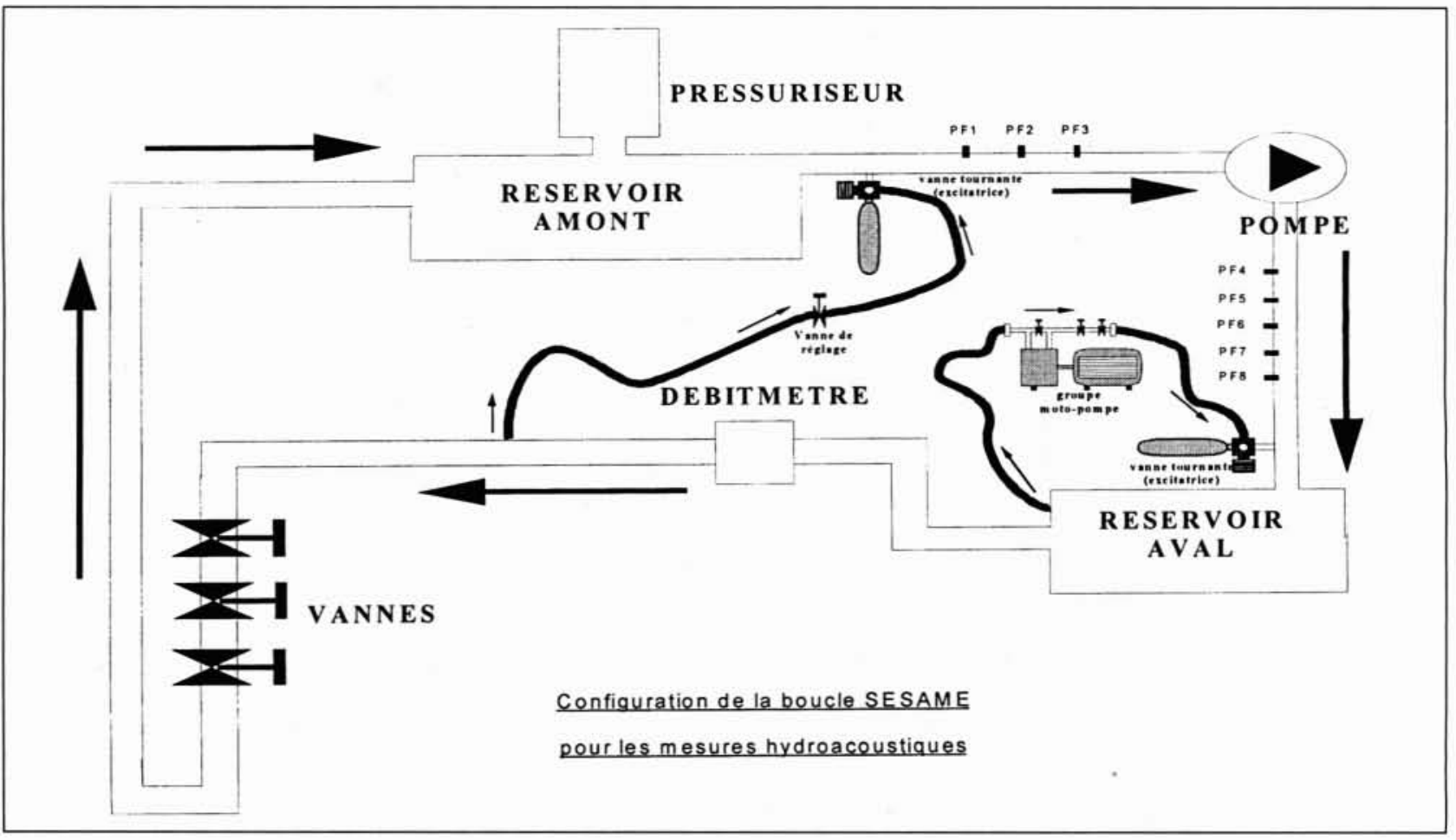

4. Schéma de la boucle SESAME. 
pées de 8 capteurs de pression fluctuante KISTLER 701A, répartis en 2 ensembles :

- 3 capteurs à l'aspiration,

- 5 capteurs au refoulement.

\section{IV — LA POMPE TESTÉE}

C'est la roue dite SHF/A2 qui a été installée sur la boucle d'essais SESAME; en voici quelques caractéristiques :

- roue centrifuge

- nombre d'aubes

- vitesse de rotation nominale

- débit nominal

- hauteur nominale

$1330 \mathrm{tr} / \mathrm{mn}$

$495 \mathrm{~m}^{3} / \mathrm{h}$

$32,8 \mathrm{~m}$

\section{V — LES ESSAIS RÉALISÉS}

Tout d'abord, lors de la quasi-totalité de ces essais, la vitesse de rotation de la pompe a été maintenue constante et égale à $1330 \mathrm{tr} / \mathrm{mn}$. Nous avons déjà étudié le paramètre vitesse de rotation lors de précédents essais, mais sur une autre pompe centrifuge. Les amplitudes des pics, pour la fréquence de rotation de la machine et pour la fréquence de passage des aubes, varient comme le carré du rapport des vitesses de rotation.

Dans le tableau ci-après sont listés tous les essais réalisés ; en plus des essais dits «normaux » à différents débits, se sont ajoutés des essais dits "particuliers " comme débit nul-pompe en rotation (essai 17) et même débit nul-roue à l'arrêt (essai 18); la position de l'aube par rapport au bec de volute (roue à l'arrêt) a aussi été étudiée (essais 22 à 24); enfin un essai dit "cavitant ", pression minimum à l'aspiration (essai 21), nous a démontré que, dans ce cas, notre méthodologie expérimentale était à la limite de sa validité.

\section{VI — LES PRINCIPAUX RÉSULTATS OBTENUS}

\subsection{Spectres}

On a représenté, sur la figure 6, un spectre à l'aspiration et un spectre au refoulement de la pompe et leurs variations en

\begin{tabular}{|c|c|c|l|}
\hline $\begin{array}{c}\text { Essai } \\
\mathrm{N}^{*}\end{array}$ & $\begin{array}{c}Q / Q n \\
(\%)\end{array}$ & $\begin{array}{c}Q \\
\left(\mathrm{~m}^{3} / \mathrm{h}\right)\end{array}$ & Remarques \\
\hline 1 & 110 & 550 & \\
\hline 2 & 100 & 495 & débit nominal \\
\hline 3 & 90 & 450 & \\
\hline 4 & 80 & 400 & \\
\hline 5 & 70 & 350 & \\
\hline 6 & 65 & 325 & \\
\hline 7 & 60 & 300 & apparition de la \\
recirculation ?
\end{tabular}

\begin{tabular}{|c|c|c|c|}
\hline $\begin{array}{l}\text { Essai } \\
\mathrm{N}^{\circ}\end{array}$ & $\begin{array}{c}Q / Q n \\
(\%)\end{array}$ & $\underset{\left(m^{3} / h\right)}{Q}$ & Remarques \\
\hline 14 & 15 & 75 & \\
\hline 15 & 10 & 50 & \\
\hline 16 & 5 & 25 & \\
\hline 17 & 0 & 0 & $\begin{array}{l}\text { débit nul (pompe } \\
\text { en rotation) }\end{array}$ \\
\hline 18 & 0 & 0 & $\begin{array}{l}\text { sans écoulement } \\
\text { (pompe à l'arrêt) }\end{array}$ \\
\hline 19 & 100 & 495 & $\begin{array}{l}\text { inversion } \\
\text { des excitatrices }\end{array}$ \\
\hline 20 & 100 & 495 & $\begin{array}{l}3 \text { cycles } \\
\text { d'excitation }\end{array}$ \\
\hline 21 & 100 & 495 & Qn, CAVITANT \\
\hline 22 & 0 & 0 & $\begin{array}{l}\text { pompe arrêt, } \\
\text { bec en face } 28^{\circ}\end{array}$ \\
\hline 23 & 0 & 0 & $\begin{array}{l}\text { pompe arrêt, bec } \\
\text { à } 1 / 3,45^{\circ}\end{array}$ \\
\hline 24 & 0 & 0 & $\begin{array}{l}\text { pompe arrêt, bec } \\
\text { à } 2 / 3,62^{\circ}\end{array}$ \\
\hline
\end{tabular}

5. Liste des essais réalisés.

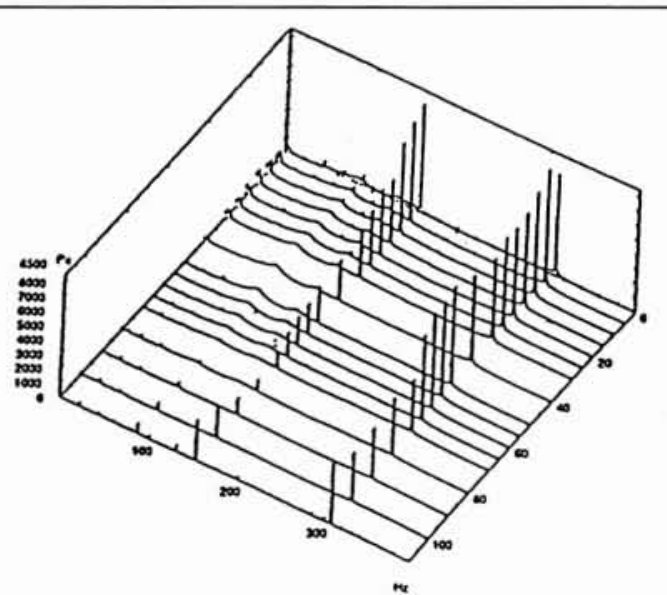

6. Spectres des fluctuations de pression en fonction du débit $(Q / Q n)$ à l'aspiration (à gauche) et au refoulement (à droite) de la pompe. 
fonction de la charge (notez la différence d'échelle afin de pouvoir représenter les pics à certains multiples de la vitesse de rotation)

A partir de $60 \%$ de la charge et pour les charges inférieures, les spectres commencent à se déformer, ce qui semble correspondre à l'apparition de la recirculation, phénomène typique des fonctionnements à régimes partiels d'une pompe.

En ce qui concerne les pics aux multiples de la vitesse de rotation, les plus importants sont ceux à la vitesse de passage des aubes et à son double $(7 \times 22,167=155 \mathrm{~Hz}$ et son double $310 \mathrm{~Hz}$ )

Leurs augmentations en fonction de la charge sont notables, et varient de manière parabolique. Notez que le minimum d'un pic n'est pas au régime nominal, mais à une charge un peu inférieure, correspondant au point de rendement maximum.

\subsection{La matrice de transfert de la pompe}

Les excitatrices (vannes 1 trou) ont été utilisées entre 7 et 45 $\mathrm{Hz}$ et ont donc bien recouvert la zone $15-90 \mathrm{~Hz}$; aux fréquences supérieures, seules les harmoniques apparaissent, d'intensité moindre, mais souvent suffisantes pour couvrir le bruit hydraulique de la machine.

Avec la méthodologie présentée précédemment, la matrice de transfert de la pompe a été établie :

- pour tous les essais à débit non nul (5\% à $110 \%)$,

- pour tous les essais à débit nul (roue en rotation ou à l'arrêt).

Pour tous les essais, les quatre termes de la matrice de transfert ne varient pas.
Sur la figure 7 , sont représentés les 4 termes de la matrice, pour le point nominal et pour les 3 essais à débit nul, roue à l'arrêt.

On constate que, surtout pour les fréquences inférieures à $90 \mathrm{~Hz}$ (zone vraiment bien recouverte par les excitatrices) : - les termes diagonaux sont très voisins de la valeur réelle 1 ,

- les termes anti-diagonaux sont quasiment imaginaires purs et représentent respectivement l'impédance $Z_{0}$ et l'admittance $1 / Z_{0}$.

De toutes ces constatations, on peut déduire que pour l'établissement, d'une manière expérimentale de la matrice de transfert d'une pompe centrifuge, un seul essai peut suffire; de plus, cet essai peut se faire sans écoulement (débit nul) et même sans faire tourner le moteur (roue à l'arrêt).

En résumé, un fabricant de pompes pourrait donc fournir, sans investissement grandiose, la matrice de transfert de sa machine à l'installateur.

\subsection{Les sources hydroacoustiques}

Par différence avec un essai sans excitatrice, les sources hydroacoustiques de la pompe ont été estimées pour les différentes charges étudiées.

Sur la figure 8 , sont représentées les 2 sources de débit, - à $100 \%$ de charge, à $60 \%$ de charge et à $10 \%$ de charge.

On constate, en ce qui concerne le bruit large bande, une nette augmentation entre $100 \%$ et $60 \%$ de charge $(10$ à 20 fois plus important) et une quasi-constance pour les charges plus faibles.

En ce qui concerne les pics visibles à certains multiples de la vitesse de rotation, leurs variations sont les mêmes que celles constatées dans le paragraphe « spectres ».

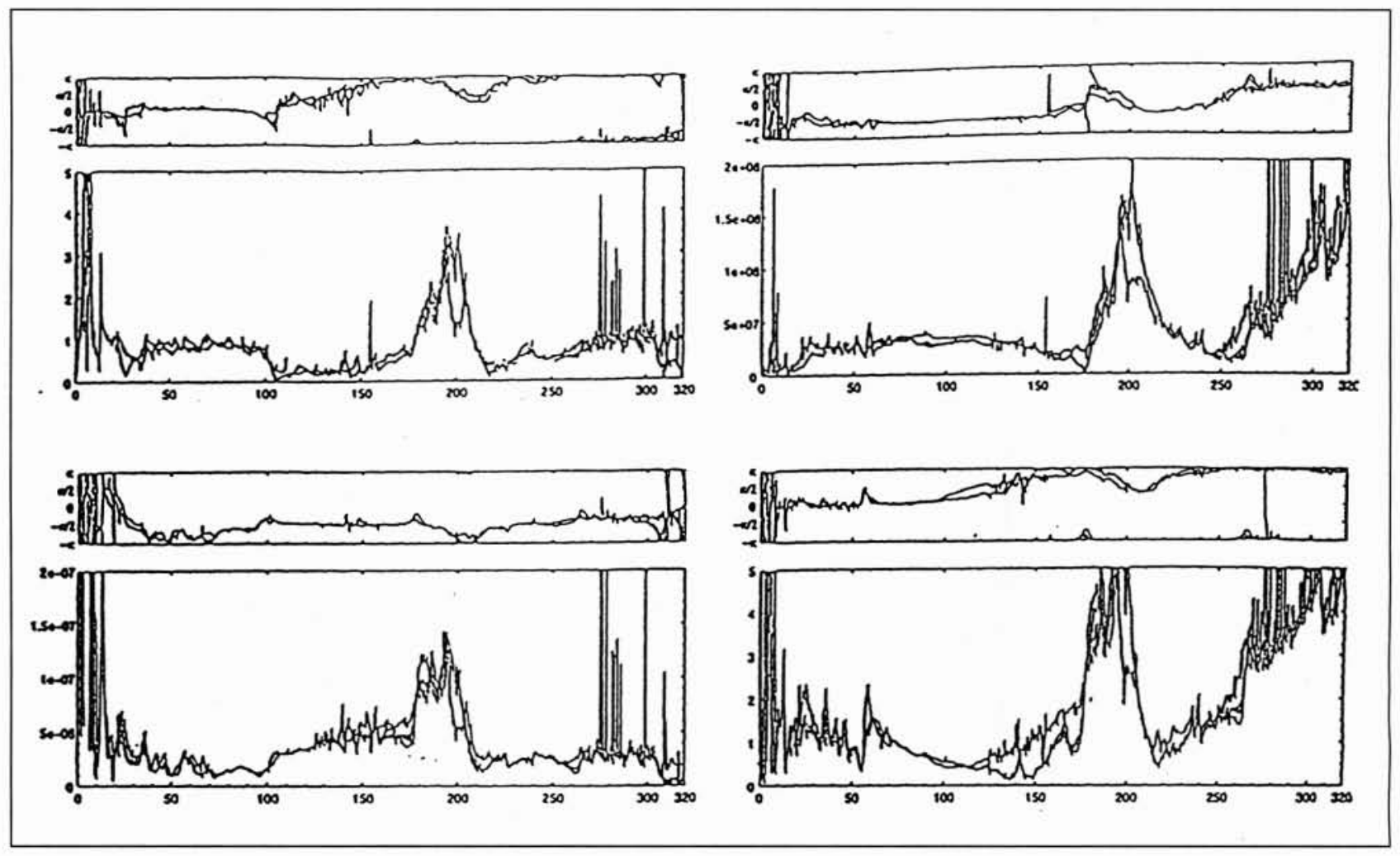

7. Les quatre termes de la matrice de transfert d'une pompe centrifuge. 


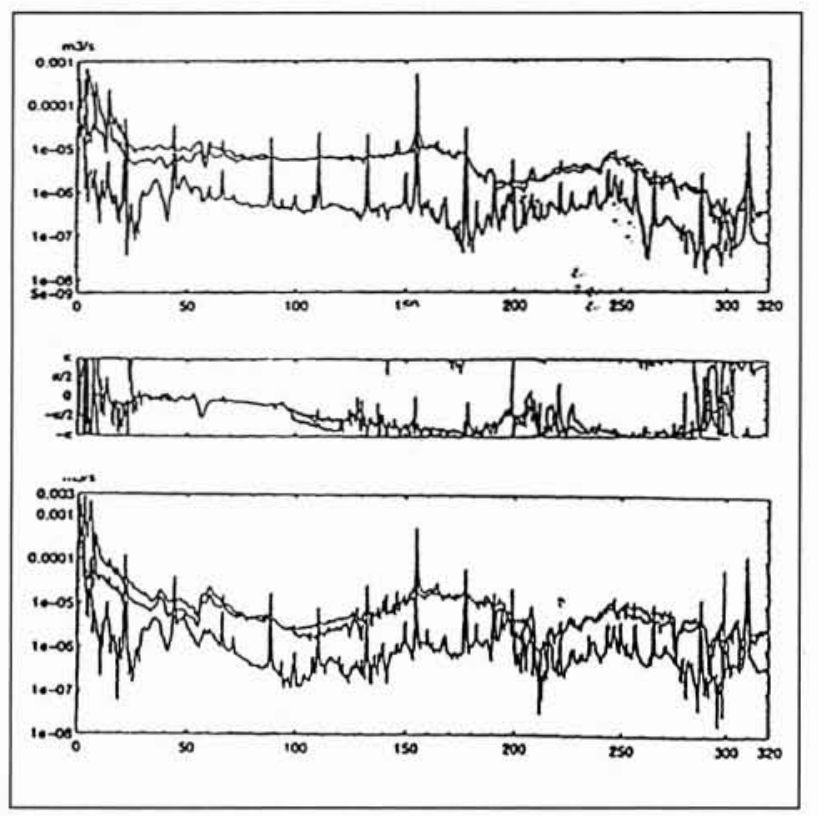

8. Les 2 sources de débit de la pompe en fonction de la charge (à l'aspiration en haut, au refoulement en bas, déphasage au milieu).

Enfin, concernant la phase, une référence a été prise au refoulement de la pompe; la source à l'aspiration est alors, ou en phase, ou bien en opposition de phase avec la source au refoulement.

\section{VII $\square$ CONCLUSION}

Cette étude est trop récente pour être complète et définitive. Les principaux résultats obtenus par l'étude expérimentale ont été présentés, mais il reste à établir, pour CIRCUS, le modèle hydroacoustique d'une pompe centrifuge à basses charges (matrice de transfert et sources acoustiques) qui se rattache d'une manière cohérente au modèle validé autour de la charge nominale.

$\mathrm{Si}$ les quatre termes de la matrice de transfert ne varient guère en fonction de la charge, les sources acoustiques évoluent (les pics et le bruit large bande) et doivent être écrites sous forme de formules simplifiées en fonction de la charge.

\section{BIBLIOGRAPHIE}

[1] Jacob Th., Trollat C. (1991), - Hydroacoustic Caracteristics of Centrifugal Pumps. IAHR-WG1 Meeting. Milan.

[2] VILlouvier V. - Détermination expérimentale de sources et de matrices de transfert acoustiques : méthodes et moyens d'essais. EDF/DER-HP-63/92.172.

[3] LAURo J.-F., Boyer A. (1993). - Hydroacoustique d'une pompe. Caractérisation des transferts d'une pompe au moyen d'une excitatrice de débit. IAHR-WG1 Meeting. Lausanne.

[4] LAURo J.-F., BOYER A. - Determination of the hydroacoustic transfer matrix and sources of a pump. An experimental method. NOISE CON 94. Fort Lauderdale (USA).

[5] Boyer A., LAURo J.-F. (1995). - Dynamic Interaction between a Pump and its Test Loop. IAHR-WG1 Meeting. Ljubljana.

[6] Trolle J.-L., Leduce D. (1995). - Comportement Vibroacoustique de Réseaux de Tuyauteries Excités par des Ecoulements. Revue Française de Mécanique $\mathrm{N}^{\circ} 2$. 